Forthcoming in Thought: A Journal of Philosophy

\title{
UNANIMOUS CONSENSUS AGAINST AGM?
}

\author{
RUSH T. STEWART
}

\begin{abstract}
Given the role consensus is supposed to play in the social aspects of inquiry and deliberation, it is important that we may always identify a consensus as the basis of joint inquiry and deliberation. However, it turns out that if we think of an agent revising her beliefs to reach a consensus, then, on the received view of belief revision, AGM belief revision theory, certain simple and compelling consensus positions are not always available.
\end{abstract}

Keywords. AGM; belief revision; consensus; disagreement; unanimity

\section{INTRODUCTION}

Concern for epistemic consensus is a hallmark of pragmatist epistemology. In "The Fixation of Belief," Peirce criticizes certain methods of belief formation on the grounds that their failure to secure wide-spread consensus will undermine the resulting beliefs (Peirce, 1992a, pp. 116-117). In other places, Peirce can be interpreted as proposing a definition of truth in terms of consensus in the long run of inquiry, or as asserting that, if inquiry were to go on indefinitely, the truth would be consensually settled upon in the limit (e.g., Peirce, 1992b, pp. 138-139; Misak, 2004, pp. 67-70). Later, and notoriously, Rorty advocated consensus-type accounts of knowledge and truth. As Guignon and Hartely summarize his view, "There is no basis for deciding what counts as knowledge and truth other than what one's peers will let one get away with in open exchange of claims, counterclaims, and reasons" (2003, p. 11). Consensus also figures prominently in Isaac Levi's brand of pragmatism. Levi denies that "inquiry can proceed without appeal to some point of view (state of full belief, demands for information, judgments of credal probability, etc.)," while also denying that "there is some standard, objective point of view to which appeal may always be made" (1991, p. 87). However, these denials are not tantamount to "cognitive licentiousness" or "epistemic anarchy," according to Levi, because we can always identify a consensus as shared agreement that can function as a non-question-begging initial position for joint inquiry (1991, pp. 87-88). On Levi's proposal, parties to a consensus should restrict themselves to the shared agreements between their points of view. Once on common ground, the group can engage in hypothetical reasoning and inquiry.

Unanimity or shared agreement is one simple and perhaps obvious account of consensus. On this view, epistemic consensus among some belief sets consists of the beliefs held in common, those beliefs that are unanimously held (Levi, 1996, Chp. 2). In a sense, shared agreement is the analogue of the conciliatory position in the literature on peer disagreement for sets of beliefs (e.g., Christensen, 2009; Feldman, 2011). A shift to consensus as shared agreement suspends judgment on beliefs about which there is not agreement. It turns out, though, that on the received view of how an agent ought to revise her beliefs, AGM belief revision theory, unanimous consensus is not always available to serve as the basis of joint inquiry or deliberation. The key move here is to think of reaching a consensus (for an agent) as an agent revising her beliefs to a belief set that represents a consensus position for some set of rival belief sets. In light of the results presented below, we are confronted with a classic modus ponens/modus tollens dilemma. On the one hand, if an agent should always be able to contract her belief set to unanimous consensus with another belief set, then the propositions that follow are damning for AGM partial meet contraction. On the other hand, if the AGM account or another account for which analogous results hold is found sufficiently

Date: September 27, 2017. 
compelling, so much the worse for the availability of unanimity for the purposes of collective inquiry and deliberation. This note establishes the existence of such a dilemma.

\section{AGM Contraction}

The unanimous consensus or consensus as shared agreement for two sets of beliefs, $K_{1}$ and $K_{2}$, is given by the beliefs common to both sets, that is, by the intersection: $K_{1} \cap K_{2}$. So, a transition from either $K_{1}$ or $K_{2}$ to $K_{1} \cap K_{2}$ must be a contraction, an operation of belief removal. How ought an agent to go about contracting her beliefs? The AGM paradigm offers perhaps the most familiar and well-explored proposal. Let's briefly review it.

Let $\mathcal{L}$ be a propositional language that is closed under truth functional operations. We let lower case Greek letters, $\alpha, \beta, \ldots$ (except $\gamma$ ), range over sentences, and capital Roman letters, $A, B, \ldots$, refer to sets of sentences. By closure, if $\alpha \in \mathcal{L}$, then $\neg \alpha \in \mathcal{L}$. If $\alpha, \beta \in \mathcal{L}$, then $\alpha \vee \beta$ is in $\mathcal{L}$, and so on. We use lower case Roman letters, $p, q, \ldots$, to denote the atomic formulae of the language. Let $C n$ be a Tarskian consequence operator: $C n: \mathscr{P}(\mathcal{L}) \rightarrow \mathscr{P}(\mathcal{L})$. A sentence $\alpha$ is in $C n(A)$ if and only if $\alpha$ is a logical consequence of $A .^{1}$ We call $K$ a theory if $K$ is deductively closed, i.e., $K=C n(K)$. Let $\mathbb{K}$ denote the set of all deductively closed subsets of $\mathcal{L}$. Belief sets are elements of $\mathbb{K}$, with sentences representing beliefs.

There are three standard belief change operations. In expansion, a sentence is added to $K$. But expansion can introduce inconsistency. Revision incorporates a sentence into $K$ while preserving consistency. Contraction removes beliefs from $K$. We put $K \dot{-\alpha}$ for the result of contracting $\alpha$ from $K$ with $-: \mathbb{K} \times \mathcal{L} \rightarrow \mathbb{K}$. There are choices to be made in contraction since, in general, how to alter $K$ so that a sentence is no longer a consequence is not uniquely determined. For example, if $\alpha, \alpha \rightarrow \beta, \beta \in K$, then at least one of the other sentences, $\alpha$ or $\alpha \rightarrow \beta$, must also be surrendered if $\beta$ is to be contracted. The AGM postulates for contraction are as follows.

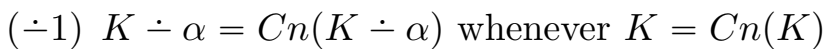

$(\dot{-} 2) K \dot{-} \alpha \subseteq K$

$(-3)$ If $\alpha \notin K$, then $K \subseteq K \dot{-} \alpha$

$(\dot{-4)}$ If $\alpha \in K \dot{-} \alpha$, then $\alpha \in C n(\varnothing)$

$(\div 5) K \subseteq C n((K \dot{-} \alpha) \cup\{\alpha\})$

$(\dot{-6)}$ If $C n(\alpha)=C n(\beta)$, then $K \dot{-\alpha}=K \dot{-} \beta$

$(\dot{-} 7) K \dot{-\alpha} \cap K \dot{-} \beta \subseteq K \dot{-}(\alpha \wedge \beta)$

$(\div 8)$ If $\alpha \notin K \dot{-}(\alpha \wedge \beta)$, then $K \dot{-}(\alpha \wedge \beta) \subseteq K \dot{-} \alpha$

The first six postulates are known as the basic postulates; the last two, as the supplementary postulates.

Various concrete contraction constructions have been explored in the literature. In partial meet contraction, the central focus is on so-called remainder sets, the set of all maximal subsets of $K$ that do not imply $\alpha$, the $\alpha$-remainders of $K$. The typical stated motivation for such focus is informational economy. Because information is valuable, agents should seek to retain as much of it as possible when contracting their belief sets. ${ }^{2}$

\footnotetext{
${ }^{1}$ As is customary, we assume $C n$ satisfy certain standard properties: (Inclusion) $A \subseteq C n(A)$

(Monotonicity) If $A \subseteq B$, then $C n(A) \subseteq C n(B)$

(Iteration) $\quad C n(A)=C n(C n(A))$

(Supraclassicality) If $\alpha$ is a classical consequence of $A$, then $\alpha \in C n(A)$

(Deduction) $\beta \in C n(A \cup\{\alpha\})$ iff $\alpha \rightarrow \beta \in C n(A)$

(Compactness) If $\alpha \in C n(A)$, then $\alpha \in C n\left(A^{\prime}\right)$ for some finite $A^{\prime} \subseteq A$

${ }^{2}$ Levi has stressed in numerous places that the loss of informational value - and not of informational content-is what should be minimized in contraction $(1991 ; 2004)$. The point here is that it is possible for $K^{\prime}$ to bear as much informational value as $K$ even if $K^{\prime} \subset K$. Consequently, restricting our attention to remainder sets is misguided. Hans Rott argues that, in any case, informational economy does not play the role in AGM belief revision that it is generally
} 
Definition 1. The set of $\alpha$-remainders of $K$ is given by

$$
K \perp \alpha=\left\{K^{\prime} \subseteq K:(i) \alpha \notin C n\left(K^{\prime}\right),(i i) \text { if } \beta \in K \text { but } \beta \notin K^{\prime} \text {, then } \beta \rightarrow \alpha \in K^{\prime}\right\}
$$

A partial meet contraction takes the intersection of some selection of elements in $K \perp \alpha$. The selection function $\gamma: \mathscr{P}(\mathbb{K}) \rightarrow \mathscr{P}(\mathbb{K})$ chooses a nonempty subset $\gamma(K \perp \alpha)$ of $K \perp \alpha$ (when $K \perp \alpha=$ $\varnothing, \gamma(K \perp \alpha)=K)$. We call - a partial meet contraction operator just in case there exists a selection function such that the following holds:

$$
K \bullet \alpha=\bigcap \gamma(K \perp \alpha)
$$

The six basic contraction postulates characterize partial meet contraction.

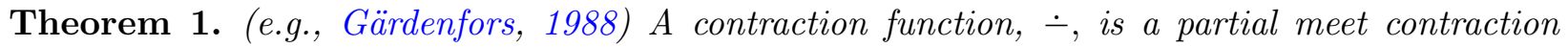

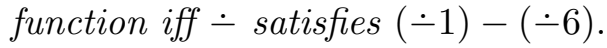

The supplementary postulates constrain the partial meet contractions further (by constraining the behavior of $\gamma$ ).

\section{Contracting to Consensus}

When two agents with consistent belief sets, $K_{1}$ and $K_{2}$, disagree on some $\delta$ in the sense that $\delta \in K_{1}$ and $\neg \delta \in K_{2}$, it is clear that each can suspend judgment on $\delta$ by contracting by $\delta$ and $\neg \delta$, respectively (Elkin, 2015). However, articulating an account of how to transition to a particular desired corpus has not been the AGM agenda. Instead, the aim is to provide an account of how to accommodate some given input (in contraction, the "input" sentence is to be removed). But the motivation or justification for the input sentence is not part of the formal account of belief revision in the AGM paradigm. It is reasonable to think that one possible motivation for contracting a particular sentence may be to achieve consensus with another corpus. That is, the desire to shift to a specific sub-corpus may underwrite contracting a particular sentence. Is focusing on a single sentence in general sufficient to reach consensus as shared agreement? Put another way, is the belief set that represents unanimous consensus with another belief set always accessible in the AGM framework?

No. We can establish the following proposition.

Proposition 1. It is not the case that, for any consistent belief sets $K_{1}, K_{2} \in \mathbb{K}$ and any partial meet contraction operator $\dot{-}$, there is a sentence $\alpha$ such that $K_{1} \dot{-} \alpha=K_{1} \cap K_{2}$.

(Proofs of propositions are relegated to the appendix.) The upshot of Proposition 1 is that there are partial meet contraction operators for which consensus with certain other belief sets is not available. We might wonder, however, about epistemically opportunistic agents. Perhaps an agent would be willing to employ a different partial meet contraction operator just to reach consensus. But much of the work in belief revision presupposes that an agent is committed to a unique contraction operator at a given time (e.g., Hansson, 2003, p. 44). Given well-known results in the literature, this is tantamount to an agent's being committed (at a given time) to a single way of assessing what the "best" elements of $K \perp \alpha$ are in the case of partial meet contraction, or a single way of assessing the epistemic usefulness or value of sentences in $\mathcal{L}$ in the case of epistemic entrenchment, or a single way of assessing, essentially, the plausibility of possible worlds in the case of a Grove system of spheres.

Furthermore, in the infinite case, there are other straightforward limitations, as the next proposition attests. Proposition 2 demonstrates that the strategy of either putting further restrictions on the class of partial meet contractions - so that only full meet or some other subset of the partial meet contractions are allowed - or permitting agents to employ alternative partial meet contraction

ascribed (2000). Consider, for example, the fact that partial meet contraction and not maxichoice contraction plays the central role. 
operators for the purpose of contracting to consensus is not a general workaround for the limitation indicated in Proposition 1.

Proposition 2. Let $\mathcal{L}$ contain infinitely many atomic sentences. It is not the case that for every pair of consistent belief sets $K_{1}, K_{2} \in \mathbb{K}$ there is some partial meet contraction, - , such that $K_{1} \doteq \alpha=K_{1} \cap K_{2}$ for some sentence $\alpha$.

So there are belief sets for which no partial meet contraction operator yields consensus for some sentential input. Proposition 2 represents more than a mere remote mathematical obstacle for AGM contraction according to a pragmatist of Levi's stripe. Pragmatists have long resisted accounts of privileged or fixed languages and conceptual schemes. We should be open to refining our language as appropriate.

We might consider equipping each belief set with a special-purpose consensus contraction operator, $\dot{ }_{K_{K_{2}}}$, such that $K_{1} \dot{ }_{C_{K_{2}}} \alpha=K_{1} \cap K_{2}$ for any $\alpha \in \mathcal{L}$. In a similar vein, Levi considers consensus-based revisions which result from first contracting to shared agreements (e.g., $K_{1} \dot{ }_{K_{K_{2}}} \alpha$ ), then adding a sentence (Levi, 1996, p. 42). Consensus-based revisions can be used to engage in group hypothetical reasoning from a shared background corpus, for example. As the next observation shows, $\dot{-}_{K_{K_{2}}}$ is at odds with the AGM vision of belief contraction (I omit the proof).

Proposition 3. Let $K_{1}, K_{2} \in \mathbb{K}$ and define $\dot{-}_{C_{K_{2}}}$ by setting $K_{1} \dot{-}_{C_{K_{2}}} \alpha=K_{1} \cap K_{2}$ for all $\alpha \in \mathcal{L}$. $\dot{-}_{K_{2}}$ satisfies $(-1),(-2),(\dot{-6}),(\dot{-} 7)$, and $(\dot{-8)}$; however, $(\dot{-3)},(\dot{-4)}$, and $(\dot{-5)}$ are not satisfied.

\section{Discussion}

If rational contraction were restricted to AGM partial meet contraction, unanimity or consensus as shared agreement would not always be available to serve as a non-question-begging position at the outset of inquiry. An agent may have no rational recourse but to beg questions against certain other parties or points of view. Whether this is (further) evidence against AGM or against the importance of unanimous consensus requires further argumentation. But there are a few concerns that should still be addressed, even if briefly. These concerns represent ways to minimize the interest of the above results.

First, one might complain about the overly conservative nature of unanimity. For approaches that attempt to assimilate reaching an epistemic consensus to voting, for instance, the presence of a single dissenter would seem insufficient grounds for excluding an otherwise unanimously accepted proposition from the consensus corpus. If complete unanimity is required for consensus, consensus is exceedingly rare, one might object. At any rate, many social and political decisions get made without it. Though I have not undertaken a defense of unanimity here, in order for the central tension of the present note to be of real interest, the unanimity conception of consensus should not be a non-starter. Whether unanimity is a nonstarter depends on the relevant function consensus is supposed to play. If that function is to avoid begging questions or to serve as a non-controversial basis for joint inquiry and deliberation - as at least it sometimes is - it is voting accounts, not unanimity, that may fail to get off the line.

Second, we might explore a more general notion of "accessibility" within the AGM paradigm. For example, we could ask about which belief states are accessible under some finite sequence of contractions instead of by just a single contraction. We cannot gloss over the notorious problem of iterated revision here (e.g., Spohn, 1988; Boutilier, 1993; Gärdenfors and Rott, 1995; Darwiche and Pearl, 1997; Hansson, 2003; Nayak et al., 2003). Briefly put, the problem is that AGM belief revision theory and a number of variant belief revision theories constrain only a single stage of belief revision. There is no account of iterated belief change in the classic AGM framework or in many of its relatives. And while various attempts to remedy this have been made (e.g., Darwiche and Pearl, 1997; Spohn, 2012), these attempts are not without substantial controversy. A compelling solution to the problem, Hansson observes, "has turned out to be very difficult to achieve" (2003, p. 42). 
For a recent overview and critical assessment of attempts to solve the problem, see (Booth and Chandler, 2016). And then there are others, like Levi, who deny that there is a serious problem of iterated belief change because they deny that there are diachronic norms of rationality (e.g., Levi, 1980, pp. 9 - 13). On such views, we cannot make general claims about rational, iterated belief change. So, an account of reaching consensus via iterated contraction awaits a compelling account of iterated contraction which so far has proved difficult to articulate and which some think is not in the offing.

More optimistically, we might think of single-shot accessibility as providing a criterion that determines with which belief states it is rational for an agent to seek consensus. While contracting to the unanimous consensus with another belief state is not possible in general, with respect to some belief states it is. In other ways, this is a pessimistic view, as it surrenders the general possibility of non-question-begging joint inquiry and deliberation.

Third, we might distinguish standards for genuinely contracting beliefs from those for hypothetically doing so (e.g., Fuhrmann and Hansson, 1994; Levi, 1996). In supposing $\alpha$ for the sake of the argument, an agent need not actually come to believe $\alpha$. Similarly, in hypothetically contracting $\alpha$, an agent need not genuinely surrender the belief. Keeping a firm grasp on the distinction between actual beliefs and hypothetical suppositions or contractions is as important in decision making as it is in epistemology. In decision making, hypothetical reasoning is used to plan for contingencies and to consider the effects of potential choices. If standards of rational contraction are uniform across actual and hypothetical revision, the observations above point to a tension for AGM and the general possibility of shifting to consensus, whether we understand seeking consensus as a matter of genuinely or hypothetically revising beliefs. If different standards of contraction are appropriate depending on whether the belief change is genuine or hypothetical, AGM and the universal availability of unanimity can't both be constraints on a given domain. Given that AGM provides no account of how the input sentence came to be the input sentence (that is, no full account of justified expansion or contraction), we might be tempted to think of AGM as a candidate account of hypothetical belief revision. In hypothetical belief revision, it may be reasonably maintained that there is no need to justify the input (Levi, 1996, pp. 6-7). Similarly, we might not be prepared to endorse a genuine shift to shared agreements. But hypothetical shifts to unanimous agreement "for the sake of the argument" are more compelling. ${ }^{3}$ However, both the AGM axioms and the universal availability of unanimity cannot be constraints on hypothetical revision.

Fourth, while AGM is indeed a very prominent account of belief revision, there are well-explored alternatives. After all, Levi, who advocates consensus as shared agreement, rejects AGM partial meet contraction in favor of a more general account that he calls mild contraction (Levi, 2004) alias severe withdrawal (Rott and Pagnucco, 1999). It is worth pointing out that an analogous result holds for mild contraction. Unanimous consensus is not always available even with mild contraction (Stewart and Levi, MS). My concern here, though, is with the tension between the AGM account of contraction and universal availability of consensus, not the details of Levi's views. However, there are accommodating generalizations of the AGM framework. It is shown in the appendix that package contraction, for example, allows us to state a straightforward and universally sufficient condition for shifting to shared agreements (Proposition 4). Fuhrmann and Hansson ask, "can it

\footnotetext{
${ }^{3}$ We need make no claim about whether $K_{1}$ represents the totality of beliefs or just those beliefs relevant to addressing a particular question. For example, $K_{1}$ might be the deductive closure of a particular collection of beliefs regarding some physical domain - a theory - aspects of which are under dispute between $K_{1}$ and $K_{2}$. In that case, urging consensus as shared agreement on the relevant issues would appear to be less immediately objectionable than urging such a conservative consensus position on the totality of beliefs. Agent 1 may only be concerned about consensus on the physical theory because agent 2 is an "epistemic peer" in that domain, for exmaple, and rest content with lingering disagreement on other topics. If an agent's whole view about the world is the relevant issue, the account of consensus is general and covers that case, too.
} 
ever be rational to engage in [package] contraction?" Seeking consensus may provide a compelling rationale.

\section{TeChnical ApPendix}

\section{Proof of Proposition 1}

Proof. (cf. Hansson, 1999, p. 135) Let $\mathcal{L}$ be based on two atomic sentences, $p$ and $q$. $\mathcal{L}$ contains sixteen non-equivalent sentences. Let $K_{1}=C n(\{p \wedge q\})$. Then, we can list the logically distinct elements of $K_{1}:\{p \wedge q, p, p \leftrightarrow q, q, p \rightarrow q, p \vee q, q \rightarrow p, \top\}$. Let $K_{2}=C n(\{p \vee q\})$. Then, $K_{1} \cap K_{2}=K_{2}$. Consider the remainder sets of $K_{1}$.

$$
\begin{array}{ll}
K_{1} \perp p \wedge q & =\{C n(\{p\}), C n(\{q\}), C n(\{p \leftrightarrow q\})\} \\
K_{1} \perp p & =\{C n(\{q\}), C n(\{p \leftrightarrow q\})\} \\
K_{1} \perp p \leftrightarrow q & =\{C n(\{p\}), C n(\{q\})\} \\
K_{1} \perp q & =\{C n(\{p\}), C n(\{p \leftrightarrow q\})\} \\
K_{1} \perp p \rightarrow q & =\{C n(\{p\})\} \\
K_{1} \perp p \vee q & =\{C n(\{p \leftrightarrow q\})\} \\
K_{1} \perp q \rightarrow p & =\{C n(\{q\})\} \\
K_{1} \perp \top & =\varnothing
\end{array}
$$

Now let $\gamma$ be such that

$$
\begin{array}{ll}
\gamma\left(K_{1} \perp p \wedge q\right) & =\{C n(\{p \leftrightarrow q\})\} \\
\gamma\left(K_{1} \perp p\right) & =\{C n(\{q\}), C n(\{p \leftrightarrow q\})\} \\
\gamma\left(K_{1} \perp p \leftrightarrow q\right) & =\{C n(\{q\})\} \\
\gamma\left(K_{1} \perp q\right) & =\{C n(\{p \leftrightarrow q\})\} \\
\gamma\left(K_{1} \perp p \rightarrow q\right) & =\{C n(\{p\})\} \\
\gamma\left(K_{1} \perp p \vee q\right) & =\{C n(\{p \leftrightarrow q\})\} \\
\gamma\left(K_{1} \perp q \rightarrow p\right) & =\{C n(\{q\})\} \\
\gamma\left(K_{1} \perp \top\right) & =\{C n(\{p \wedge q\})\}
\end{array}
$$

With the exception of $K_{1} \dot{-p}$, the partial meet contractions are given by the sole members of the selection sets. $K_{1} \dot{-}=\bigcap \gamma\left(K_{1} \perp q\right)$, for instance, is $C n(\{p \leftrightarrow q\})$. For $K_{1} \dot{-} p$, we have $\bigcap\{C n(\{q\}), C n(\{p \leftrightarrow q\})\}=C n(\{p \rightarrow q\})$. So, not only do we have $K_{1} \dot{-} p \vee q \neq K_{1} \cap K_{2}$, but $K_{1} \dot{-\alpha} \neq K_{1} \cap K_{2}$ for any $\alpha$. In this example, $\dot{-}$ is not a maxichoice contraction. Another example sufficient to illustrate the proposition could be constructed by selecting a single element of $K_{1} \perp p$, say $p \leftrightarrow q$, converting - into a maxichoice contraction (which would also be a transitively relational partial meet contraction), but the present example illustrates that the limitations for shifting to consensus apply to partial meet contractions more generally.

\section{Proof of Proposition 2}

Proof. Let $K_{1}$ be a maximal consistent subset of $\mathcal{L}$, i.e., for any $\alpha \in \mathcal{L}$, either $\alpha \in K_{1}$ or $\neg \alpha \in K_{1}$ (but not both). Let $K_{2}=C n(\varnothing)$. We show that the full meet contraction of $K_{1}$ by any sentence $\alpha$-i.e., $\gamma\left(K_{1} \perp \alpha\right)=K_{1} \perp \alpha$, so $\bigcap \gamma\left(K_{1} \perp \alpha\right)=\bigcap K_{1} \perp \alpha$-is not identical to $C n(\varnothing)$. Specifically, we show that any full meet is a superset of $C n(\varnothing)$. And since any sentence in the full meet is also in any partial meet, it follows that no partial meet contraction of $K_{1}$ is identical to $C n(\varnothing)=K_{1} \cap K_{2}$.

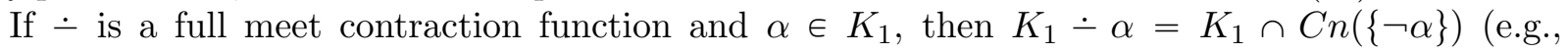
Gärdenfors, 1988, Lemma 4.9; Hansson, 1999, Observation 2.12). $\alpha$ contains instances of only finitely many atomic sentences, where $\left\{p_{i}: i \in I\right\}$ is the set of atomic sentences of $\mathcal{L}$. Let $p_{i}$ be an atomic sentence in $K_{1}$ that does not occur in $\alpha$. Clearly, $\neg \alpha \vee p_{i} \in K_{1} \cap C n(\{\neg \alpha\})$. If $\alpha \in C n(\varnothing)$, then $K_{1} \cap C n(\{\neg \alpha\})=K_{1} \neq C n(\varnothing)$. So, let $\alpha$ be contingent. Since $p_{i}$ is atomic, $p_{i} \notin C n(\varnothing)$. 
But since all of the atomic sentences are independent and $p_{i}$ does not occur in $\alpha, \neg \alpha \vee p_{i} \notin C n(\varnothing)$ either (consider the truth table). So $K_{1} \cap K_{2}=C n(\varnothing) \subset K_{1} \doteq \alpha$ for any sentence $\alpha$.

\section{Unanimous Consensus for Package Contraction}

Motivated by the limitations of "singleton" contraction, Fuhrmann and Hansson study multiple contractions, operations simultaneously contracting a set of sentences (1994). They identify two primary types of multiple contractions. Package contractions remove all of a set of sentences from the belief set; choice contractions remove at least one among a set of sentences. As they argue, multiple contraction should not be thought of in terms of sequential contraction. In sequential contractions, the order of contractions makes a difference to the resulting belief state. So a privileged sequence would have to be identified. Such a sequence finds no analogue in multiple contraction. This is to say nothing of the infamous problems confronting AGM regarding iterated revisions.

The setup for partial meet package contraction mirrors that of AGM partial meet contraction. We make use of a multiple conclusion relation, $\vdash$, familiar from sequent calculus. For sets of sentences, $A, B$, we put $A \vdash B$ when $B \cap C n(A) \neq \varnothing$. The postulates below make use of a notion of equivalence-according-to- $K . A \equiv_{K} B$ holds iff $\forall C \subseteq K: C \vdash A \Leftrightarrow C \vdash B$.

Fuhrmann and Hansson's postulates are as follows.

$(-1) K-[A] \subseteq K$

$(-2)$ If $\varnothing \nvdash A$, then $A \cap(K-[A])=\varnothing$

$(-3)$ If $A \equiv_{K} B$, then $K-[A]=K-[B]$

(-4) If $\alpha \in K \backslash K-[A]$, then there is some $K^{\prime}$ such that $K-[A] \subseteq K^{\prime} \subseteq K$ and $K^{\prime} \nvdash A$ and $K^{\prime}, \alpha \vdash A$

Definition 2. The set of all package $A$-remainders of $K$ is given by

$$
K \perp A=\left\{K^{\prime} \subseteq K:(i) K^{\prime} \forall A,(i i) \forall K^{\prime \prime}: K^{\prime} \subset K^{\prime \prime} \subseteq K \Rightarrow K^{\prime \prime} \vdash A\right\}
$$

A package partial meet contraction takes the intersection of some selection of elements in $K \perp A$

$$
K-[A]=\bigcap \gamma(K \perp A)
$$

Fuhrmann and Hansson's postulates characterize package partial meet contraction.

Theorem 2. (Fuhrmann and Hansson, 1994, Thm. 9) An operation - is a $\perp$ partial meet contraction iff - satisfies $(-1)-(-4)$ for all $K \in \mathbb{K}$ and all sets $A, B \in \mathscr{P}(\mathcal{L})$.

Proposition 4. Let - be a package partial meet contraction operator. If $A=K_{1} \backslash K_{2}$, then $K_{1}-$ $[A]=K_{1} \cap K_{2}$.

Proof. The proof proceeds by showing that $K_{1} \cap K_{2}$ is the only A-remainder of $K_{1}$, i.e., $K_{1} \perp A=$ $\left\{K_{1} \cap K_{2}\right\}$. First, then, we show that $K_{1} \cap K_{2} \in K_{1} \perp A$. It is clear that both $\left(K_{1} \cap K_{2}\right) \subseteq K_{1}$ and that $K_{1} \cap K_{2} \nvdash A$. We need only show that for any $K^{\prime} \in \mathbb{K}$, if $\left(K_{1} \cap K_{2}\right) \subset K^{\prime} \subseteq K_{1}$, then $K^{\prime} \vdash A$. Suppose the antecedent holds. Then, there is some $\alpha \in K^{\prime}$ such that $\alpha \notin K_{1} \cap K_{2}$. It follows that $\alpha \in K_{1} \backslash K_{2}=A$. Hence, $K^{\prime} \vdash A$. So, $K_{1} \cap K_{2} \in K_{1} \perp A$.

Assume for reductio that there is a $K^{\prime} \in K_{1} \perp A$ such that $K^{\prime} \neq K_{1} \cap K_{2}$. So either there is some $\alpha \in K^{\prime} \backslash\left(K_{1} \cap K_{2}\right)$, or else there is some $\alpha \in\left(K_{1} \cap K_{2}\right) \backslash K^{\prime}$. Suppose the former. Since $K^{\prime} \subseteq K_{1}, \alpha$ must be in $A$. So, $K^{\prime} \vdash A$, contrary to our assumption that $K^{\prime}$ is an $A$-remainder of $K_{1}$. Suppose the latter. Then, the only possibility is that $K^{\prime} \subset\left(K_{1} \cap K_{2}\right) \subseteq K_{1}$ (since $K^{\prime} \phi\left(K_{1} \cap K_{2}\right)$ entails that there is an $\beta \in K^{\prime} \backslash\left(K_{1} \cap K_{2}\right)$, and, again, $\beta \in K^{\prime} \backslash\left(K_{1} \cap K_{2}\right)$ entails $\left.K^{\prime} \vdash A\right)$. But clearly, $K_{1} \cap K_{2} \nvdash A$, again, contrary to our assumption that $K^{\prime}$ is an $A$-remainder of $K_{1}$. 


\section{REFERENCES}

Booth, R. and J. Chandler (2016). The irreducibility of iterated to single revision. Journal of Philosophical Logic, Forthcoming.

Boutilier, C. (1993). Revision sequences and nested conditionals. In IJCAI, Volume 93, pp. 519-531.

Christensen, D. (2009). Disagreement as evidence: The epistemology of controversy. Philosophy Compass 4(5), 756-767.

Darwiche, A. and J. Pearl (1997). On the logic of iterated belief revision. Artificial Intelligence $89(1), 1-29$.

Elkin, L. (2015). An epistemically modest response to disagreement, agm-ified. The Reasoner 9(9), $76-77$.

Feldman, R. (2011). Reasonable religious disagreements. In A. Goldman and D. Whitcomb (Eds.), Social Epistemology: Essential Readings, Volume 137. Oxford University Press.

Fuhrmann, A. and S. O. Hansson (1994). A survey of multiple contractions. Journal of Logic, Language and Information 3(1), 39-75.

Gärdenfors, P. (1988). Knowledge in Flux: Modeling the Dynamics of Epistemic States. MIT Press, Cambridge, MA.

Gärdenfors, P. and H. Rott (1995). Handbook of Logic in Artificial Intelligence and Logic Programming: Epistemic and temporal reasoning, Volume 4, Chapter Belief Revision. Oxford University Press, Oxford.

Guignon, C. and D. R. Hiley (2003). Introduction: Richard rorty and contemporary philosophy. In C. Guignon and D. R. Hiley (Eds.), Richard Rorty and Contemporary Philosophy, pp. 1-40. Cambridge: Cambridge University Press.

Hansson, S. O. (1999). A Textbook of Belief Dynamics: Theory Change and Database Updating, Volume 11. Springer Science \& Business Media.

Hansson, S. O. (2003). Ten philosophical problems in belief revision. Journal of Logic and Computation 13(1), 37-49.

Levi, I. (1980). The Enterprise of Knowledge. MIT Press, Cambridge, MA.

Levi, I. (1991). The Fixation of Belief and Its Undoing: Changing Beliefs through Inquiry. Cambridge University Press.

Levi, I. (1996). For the Sake of the Argument: Ramsey Test Conditionals, Inductive Inference and Nonmonotonic Reasoning. Cambridge University Press.

Levi, I. (2004). Mild Contraction: Evaluating Loss of Information Due to Loss of Belief. Oxford University Press.

Misak, C. (2004). Truth and the End of Inquiry: A Peircian Account of Truth. Oxford: Clarendon Press.

Nayak, A. C., M. Pagnucco, and P. Peppas (2003). Dynamic belief revision operators. Artificial Intelligence 146(2), 193-228.

Peirce, C. S. (1992a). The fixation of belief. In N. Houser and C. Kloesel (Eds.), The Essential Peirce, Volume 1: Selected Philosophical Writings (1867-1893), pp. 109-123. Indiana University Press.

Peirce, C. S. (1992b). How to make our ideas clear. In N. Houser and C. Kloesel (Eds.), The Essential Peirce, Volume 1: Selected Philosophical Writings (1867-1893), pp. 124-141. Bloomington: Indiana University Press.

Rott, H. (2000). Two dogmas of belief revision. The Journal of Philosophy, 503-522.

Rott, H. and M. Pagnucco (1999). Severe withdrawal (and recovery). Journal of Philosophical Logic 28(5), 501-547.

Spohn, W. (1988). Ordinal conditional functions: A dynamic theory of epistemic states. In W. L. Harper and B. Skyrms (Eds.), Causation in Decision, Belief Change, and Statistics, Volume 42 of The University of Western Ontario Series in Philosophy of Science, pp. 105-134. Kluwer 
Academic Publishers.

Spohn, W. (2012). The Laws of Belief: Ranking Theory and Its Philosophical Applications. Oxford University Press, USA.

Stewart, R. T. and I. Levi (MS). Consensus does not justify contraction. Unpublished Manuscript. 